\section{NDCX-II project commencing at LBNL}

Alex Friedman, LLNL, and Joe Kwan, LBNL

Construction is beginning on the second generation Neutralized Drift Compression eXperiment (NDCX-II), a new high-current, modest-kinetic-energy accelerator at Lawrence Berkeley National Laboratory (LBNL). The machine's ion beams will enable studies of the poorly understood "warm dense matter" regime of temperatures around 10,000 K and densities near solid (as in the cores of giant planets). NDCX-II will also enable exploration of important issues in inertialfusion target physics. These studies support the ultimate goal of using ion beams to heat deuterium/tritium fuel to ignition in a future inertial fusion power reactor (a role for which accelerators appear well suited). NDCX-II has received \$11 M of funding from the American Recovery and Reinvestment Act; construction began in July, 2009, with completion of the initial 15-cell configuration anticipated in March, 2012.

NDCX-II will accelerate a beam of 30-50 nanoCoulombs of $\mathrm{Li}^{+}$ions to $1.5-4 \mathrm{MeV}$ and compress it into a nanosecond long pulse. The short, high-current pulse is important for applications requiring efficient stopping of ions for rapid heating of a small amount of matter. As with the existing NDCX-I, the new machine uses neutralized drift compression. In this process, the beam's tail is given a higher velocity than its head, so that it shortens while it drifts in a plasma that provides electrons to cancel space-charge forces.

The figure shows the layout of the machine. It will make extensive use of induction cells (accelerating elements) and other parts from the decommissioned Advanced Test Accelerator (ATA) at Lawrence Livermore National Laboratory (LLNL). It will be extensible and reconfigurable; in the configuration that has received the most emphasis, each pulse will deliver $\mathrm{Li}^{+}$ions at $3 \mathrm{MeV}$ into a mm-diameter spot onto a thin-foil target. Pulse compression to $\sim 1 \mathrm{~ns}$ begins in the accelerator and finishes in the drift compression line.

NDCX-II employs novel beam dynamics to achieve unprecedented rapid pulse compression in a short ion accelerator. The $200 \mathrm{kV}$ charged transmission line pulsedpower voltage sources from ATA, called "Blumleins", can provide voltage pulses that are not longer than $70 \mathrm{~ns}$. These are shown as blue cylinders in the figure. For them to be usable, it is necessary to reduce the ion bunch duration from its initial $500 \mathrm{~ns}$. This shortening is accomplished in an initial stage of non-neutral drift compression, downstream of the injector and the first few induction cells (note the spaces between induction cells at the left end of the figure). Long-pulse voltage generators are used at the front end; Blumleins power the rest of the acceleration.

Extensive particle-in-cell computer simulation studies have enabled an attractive physics design that meets the stringent cost goal. Snapshots from a simulation video are shown in the figure. Studies on a dedicated test stand are examining the ATA hardware and supporting the development of new pulsed solenoids that will provide transverse beam confinement.

Applications of this facility will include studies of warm dense matter using uniform, volumetric ion heating of thin foil targets, and studies of ion energy coupling into an expanding plasma (such as that which occurs in an inertial fusion target). NDCX-II will also enable a better understanding of space- charge-dominated ion beam dynamics, and of beam behavior in a plasma. The machine will complement facilities at GSI in Darmstadt, Germany, but will employ lower ion kinetic energies and commensurately shorter stopping ranges in matter.

NDCX-II will contribute to the long-term goal of electric power production via "Heavy-Ion inertial Fusion." In inertial fusion, a target containing fusion fuel is heated by energetic driver beams, and undergoes a miniature thermonuclear explosion. The largest inertial confinement facility is Livermore's National Ignition Facility (NIF). NIF is expected to establish the fundamental feasibility of fusion ignition on the laboratory scale. Heavy ion accelerators offer efficient conversion of input power into beam energy, are long-lived, and can use magnetic fields for final focusing onto a target. These attributes make them attractive candidates for a power plant. The beams in such a system will require manipulations similar to those being pioneered on NDCX-II.

NDCX-II is sponsored by the U.S. Department of Energy's Office of Fusion Energy Sciences. It is being developed by a collaboration known as the Virtual National Laboratory for Heavy Ion Fusion Science (HIFS-VNL), including LBNL, LLNL, and the Princeton Plasma Physics Laboratory (PPPL).
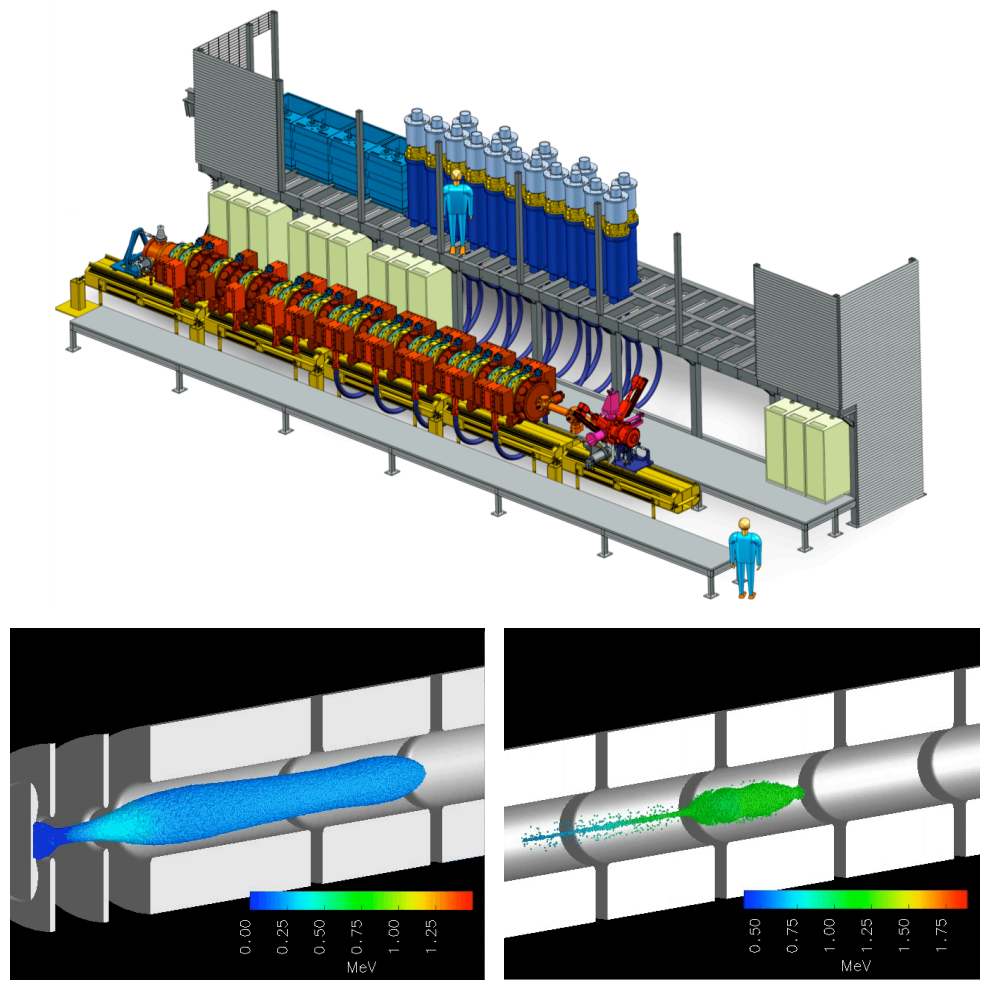

Computer-aided-design of NDCX-II, and simulation of its ion beam. The ion source and injector are at left; voltage sources (blue) reside on a mezzanine; the induction cells are in yellow-orange; and the driftcompression line and target chamber are at right. The lower images (from a 3-D simulation video using the Warp code) show the beam at the injector and farther on, undergoing inductive acceleration.

\section{Further reading}

A recent article in the Berkeley Lab News is available at http://newscenter.lbl.gov/feature-stories/2009/10/14/warm-dense-

matter; see also references therein. The video can be viewed at http://hifweb.lbl.gov/public/movies/ICAP09.

Joe Kwan is the NDCX-II project manager and Alex Friedman is the leader for the physics design. 